\title{
ESS, population games, replicator dynamics: dynamics and games if not dynamic games
}

\author{
Pierre Bernhard
}

July 21, 2008

\begin{abstract}
We review some classical definitions and results concerning Evolutionarily Stable Strategies (E.S.S.) with special emphasis with their link to Wardrop equilibrium and on the nonlinear case where the fitness accrued by an individual depends nonlinearly on the state of the population. On our way, we provide a simple criterion to check that a linear finite dimensional Wardrop equilibrium satisfies the second order E.S.S. condition. We also investigate a bifurcation phenomenon in the replicator equation associated to a population game. Finally we give two non trivial examples of Wardrop equilibria in problems where the strategies are controls in a dynamic system.
\end{abstract}

\section{Introduction}

Wardrop equilibrium, E.S.S. and related concepts form the game theoretic basis of the investigation of population dynamics under evolution or learning behaviors as depicted by the replicator equation or more generally adaptive dynamics.

Pioneered by John Glenn Wardrop in the context of road traffic as far back as 1952, [20] these concepts have been been given a new impetus after their rediscovery and extension by John Maynard-Smith and co-workers in the mid seventies, $[11,9]$ in the context of theoretical biology and evolution theory. The intoduction by Taylor and Jonker [16] of the replicator equation gave its solid mathematical grounds to the intuition of stability present from the inception. Since then a large body of literature has appeared under the generic name of evolutionary game theory. See e.g. [21, 8, 6, 17]. Nowadays, routing problems have become a hot topic again with the advent of the INTERNET and, more recently, ad hoc networks, together with learning in populations. Old topics such as optimal transportation [3] have been renewed by these new problems. All share the characteristic that they invetsigate the collective effect of rational, usually selfish, behaviour of individual 
agents on a large population of similar ones. We actally witness the emergence of a new population dynamics paradygm, where games are one of the underlying fundamental tools.

The aim of this paper is to better disseminate those ideas in the traditional Dynamic Games community, sressing among other things the links with transportation theory, and may be offering some new results on our way.

\section{Wardrop equilibrium and E.S.S.}

Let us recall the foundations of Evolutionary Stable Equilibrium theory, and in that process define our notation.

\subsection{Generating function and fitness function}

\subsubsection{Population and strategy distribution}

A large population of identical agents interact with each other. Each has the choice of several strategies $x \in X$ chosen in a set $X$. The nature of the set $X$ is an important feature of the theory. In the first part of this article, we consider the cases $X=\left\{x_{1}, x_{2}, \ldots, x_{n}\right\}$ finite or $X \subset \mathbb{R}^{n}$ a compact subset of $\mathbb{R}^{n}$. The notation $\mathrm{d} x$ will mean the Lebesgue measure if $X$ is continuous and the discrete measure if it is finite, so that in the later case,

$$
\int_{X} f(x) \mathrm{d} x=\sum_{i=1}^{n} f\left(x_{i}\right) .
$$

In the last part, we shall consider infinite dimensional $X$ spaces.

We are interested in the share or proportion of agents using each stategy, say $p(x)$. Technically, $p$ is a positive measure of mass one over $X$, and can therefore be viewed as a probability measure. We let $\Delta(X)$ denote the set of such probability measures over $X$. It is finite dimensional if and only if $X$ is finite. The measure $p(A)=\int_{A} p(\mathrm{~d} x)$ of a subset $A \subset X$ is the probability that an agent picked "at random" with uniform probability over the population use a strategy of the subset $A$ of stategies. Then the mean value of a function $f$ is

$$
\mathbb{E}^{p} f=\langle p, f\rangle=\int_{X} f(x) p(\mathrm{~d} x) .
$$

If $X$ is finite, we shall let $p\left(x_{i}\right)=: p_{i}$, we identify the measure $p$ with the vector of $\mathbb{R}^{n}$ with components $p_{i}$, and any scalar function $f$ over $X$ as the vector 
with components $f_{i}=f\left(x_{i}\right)$. Then $p$ belongs to the simplex $\Sigma_{n} \subset \mathbb{R}^{n}$ identified with $\Delta(X)$, and

$$
\mathbb{E}^{p} f=\langle p, f\rangle=\sum_{i=1}^{n} p_{i} f\left(x_{i}\right) .
$$

In evolutionary biology, the various "strategies" correspond to differences in behaviours, or "phenotypes", generated by differences in the genotypes.

The biological literature contains interesting discussions of the difference between the set up we just described, considered a genetic polymorphism, and a population, say with a finite set $X$ of possible behaviours, where all agents have the same genotype, dictating a probabilistic — or mixed - choice of behaviour, so that they all have the same probabilities $p_{i}$ of behaving according to "strategy" $x_{i}$, for $i=1,2, \ldots, n$. As a result, due to the law of large numbers, in such a genetically monomorphic population, the proportion of animals adopting the behaviour $x_{i}$ at each instant of time will be $p_{i}$, although the individuals using each $x_{i}$ may vary over time.

While these discussions are highly significant in terms of biologic understanding - e.g. some mixtures might be impossible to produce with a genetically monomorphic population, or the transmission of the behaviour over generations may be different in both cases, the more so if sexual reproduction is involved-we shall not be concerned with them. The populations are statistically the same in both cases. As a consequence, their dynamics will be considered the same. ${ }^{1}$

\subsubsection{Fitness and the generating function}

The various possible strategies induce various benefits to their users. It is assumed all along that

(i). There exists a scalar, real, measure of reward for each participant. In evolutionary biology, this measure, called "fitness", may be reproductive efficiency - the excess of the number of birth over the number of death per animal per time unit. In the example of road tafic, it will be the opposite of the time spent to reach its destination, etc.

(ii). This reward is a function of the stategy used by the particular agent and the state of the population, the later entering only through the proportions, the measure $p$.

\footnotetext{
${ }^{1} \mathrm{~A}$ Difference in the stability analysis mentioned by [10] is due to a questionable choice of dynamics in the discrete time case which we will not follow.
} 
Hence, an agent using strategy $x$ in a population characterized by a distribution of strategies $p$ has a reward - a fitness- $G(x, p)$. We call $G$ the generating function. It is assumed measurable in $x$, uniformly bounded and and weakly continuous in $p$. We shall also use the notation $G(p)$ to mean the function $x \mapsto G(x, p)$, hence in the finite case, the vector with components $G_{i}(p)=G\left(x_{i}, p\right), i=1, \ldots, n$.

A special case of interest is the linear case where $p \mapsto G(x, p)$ is linear. This is the case if, say, there is a reward $H(x, y)$ to an agent of type $x$ meeting an agent of type $y$, and "meeting" happens at random with uniform probability, so that the avarage fitness of an agent of type $x$ is

$$
G(x, p)=\int_{X} H(x, y) p(\mathrm{~d} y) .
$$

An individual with a mixed strategy $q$, or equivalently a sub-population with a strategy distibution $q$, in a population of overall distribution $p$ will get an avarage fitness

$$
F(q, p)=\langle q, G(p)\rangle=\int_{X} G(x, p) q(\mathrm{~d} x) .
$$

It follows from this definition that $F$ is always linear with respect to its first argument, but not necessarily with respect to the second one. We shall refer to the linear case to mean linearity of $G$ w.r.t. $p$, hence of $F$ w.r.t. its second argument.

\subsection{Stable population states}

\subsubsection{Wardrop equilibrium}

Consider a population with distribution $p$. Assume that in that population, some individuals mutate, creating a small sub-population of total relative mass $\varepsilon$ and of distibution $q$. The overall population now has a distribution

$$
q_{\varepsilon}=\varepsilon q+(1-\varepsilon) p .
$$

We say that the sub-population invades the original population if ${ }^{2} F\left(q, q_{\varepsilon}\right) \geq$ $F\left(p, q_{\varepsilon}\right)$. The original population will be considered evolutionarily stable if it is protected against invasion by any (single ${ }^{3}$ ) mutation. We therefore state:

Definition 1 A distribution $p$ is an Evolutionarily Stable Strategy (E.S.S.) if

$$
\forall q \in \Delta(X), \exists \varepsilon_{0}: \forall \varepsilon \leq \varepsilon_{0}, \quad F\left(q, q_{\varepsilon}\right)<F\left(p, q_{\varepsilon}\right) .
$$

\footnotetext{
${ }^{2}$ As mathematicians, we take this as our axiomatic definition of invading. The relationship to the biological concept as well as the choice of a large inequality here is a discussion left to the biologists. Our convention here is that of the biological literature [10]

${ }^{3}$ The case of simultaneous mutations is more complex.
} 
It follows from the dominated convergence theorem that the fitness function $F$ inherits the continuity of $G$ with respect to its second argument. Therefore, letting $\varepsilon$ go to zero, one immediately sees that a necessary condition for $p$ to be an E.S.S. is that

$$
\forall q \in \Delta(X), \quad F(q, p) \leq F(p, p) .
$$

J-G. Wardrop [20] was considering a road network and a population of drivers in a road network. Their strategy is the choice of a route in the network. The time of travel is a function of the occupation of the route chosen. And if the total population is fixed, this is a function of the proportion $q_{i}$ of that population that uses the route considered. We quote from [20]:

Consider the case of a given flow of traffic $Q$ which has the choice of $D$ alternative routes from a given origin to a given destination, numbered $1,2, \ldots D .[\ldots]$ Consider two alternative criteria based on these journey times which can be used to determine the distribution on the routes, as follows

(i). The journey times on all routes actually used are equal, and less than those which would be experienced by a single vehicle on any unused route.

(ii). The average journey time is minimum.

The first criterion is quite a likely one in practice, since it might be assumed that traffic will tend to settle down into an equilibrium situation in which no driver can reduce his journey time by choosing a new route.[...]

Notice that the argument developed is one of stability. It should be further noticed that his "generating function", the travel time on a route, is nonlinear, of the form $t_{i}=b_{i} /\left(1-q_{i} / p_{i}\right)$ for constants $b_{i}$ and $p_{i}$ that depend on the network and the rest of its usage. ${ }^{4}$

Extending Wardrop's definition to an infinite set $X$, we end up with the Wardrop condition, which is equivalent to, condition (4):

$$
\begin{aligned}
& \forall x \in X, \quad G(x, p) \leq F(p, p), \\
& \text { Let } Z(p)=\{x \mid G(x, p)<F(p, p)\}, \quad \text { then } \quad p(Z(p))=0 .
\end{aligned}
$$

\footnotetext{
${ }^{4}$ Alain Haurie has pointed out that the phrase "Wardrop equilibrium" now means the state of a transportation network where the traffic flow satisfies this condition for all pairs origin-destination actually served. Wardrop explicitly considers a single pair origin-destination. However, it is clear that, on the one hand, he meant it to hold for all, and on the other hand, a simple dynamic programming type of reasoning shows that if a flow is in such an equilibrium for one pair of origin-destination, it is in equlibrium for any such pair served by that flow.
} 
In recognition of Wardrop's anteriority, and following the standard terminology in routing theory, we let

Definition 2 A distribution p satisfying (4), or equivalently (5), is called a Wardrop equilibrium.

Link with Nash equilibrium One may notice that (4) is equivalent to stating that $(p, p)$ is a (symmetric) Nash equilibrium of the two player-game where the rewards $J^{1}$ and $J^{2}$ of the players are $J^{1}\left(q^{1}, q^{2}\right)=F\left(q^{1}, q^{2}\right), J^{2}\left(q^{1}, q^{2}\right)=F\left(q^{2}, q^{1}\right)$. As a consequence most of the literature on evolutionary games uses the phrase "Nash equilibrim" where we follow the usage of the transportation literature with "Wardrop equilibrium". Recognizing a Nash equlibrium makes the equivalence of (4) and (5) a trivial extension of Von Neumann's equalization theorem [18, 19], although in the biology literature, it is sometimes attributed to ([5]).

\subsubsection{Second order E.S.S. condition}

Not all Wardrop equilibria are E.S.S., because (4), or equivalently (5), is only a necessary condition for (3) to hold. Let the best response map $B(\cdot)$ be defined as

$$
B(q)=\left\{r \in \Delta(X) \mid F(r, q)=\max _{r \in \Delta(X)} F(r, q)\right\} .
$$

Notice that $B(p)$ is precisely the set of distributions $q$ that satisfy $q(Z(p))=0$.

Proposition 1 In the linear case, a Wardrop equilibrium $p$ is an E.S.S. if and only if

$$
\forall q \in B(p), \quad F(p, q)>F(q, q) .
$$

or equivalently

$$
\forall q \in B(p), \quad F(q-p, q-p)<0 .
$$

proof If $q \notin B(p)$, then by definition (3) is satisfied. If $q \in B(p)$, in the inequality

$$
F\left(q, q_{\varepsilon}\right)-F\left(p, q_{\varepsilon}\right)<0,
$$

write either $F\left(r, q_{\varepsilon}\right)=(1-\varepsilon) F(r, p)+\varepsilon F(r, q)$ or $F(r, q)=F(r, p)+\varepsilon F(r, q-p)$ with both $r=q$ and $r=p$, and use the linearity of $F$ and the fact that $F(q, p)=$ $F(p, p)$ to get the form (6) or (7) respectively.

Notice that $F(r, r)$ is a quadratic form. Therefore this last condition clearly shows that the second E.S.S. condition (6) is in fact a second order condition.

We stay for the time being with the linear case. Let a Wardrop equilibrium $p$ be fixed, and $X_{1}(p)=X-Z(p)$, the set of measure one for distributions in 
$B(p)$. Let $H_{1}$ be the restriction of $H$ to $X_{1}(p) \times X_{1}(p)$, and $F_{1}$ the corresponding bi-linear function for measures (not necessarily positive) over $X_{1}(p)$. Let also $\mathbb{1}$ be the constant function equal to one over $X$ (or over $X_{1}(p)$ ); and notice that $p-q$ in (7) is orthogonal to $\mathbb{1}$ in the space $\mathcal{M}\left(X_{1}\right)$ of measures over $X_{1}$. Let also $X_{2}$ be the support of $p$. From Wardrop's condition, it follows that $X_{2} \subset X_{1}$. Define similarily the restriction $H_{2}$ of $H$ to $X_{2} \times X_{2}$ and $\mathcal{M}\left(X_{2}\right)$. We therefore have ${ }^{5}$ :

Theorem 1 In the linear case, a Wardrop equilirium $p$ is an E.S.S.

(i). if the restriction of the quadratic form $F_{1}(r, r)$ to measures $r \in \Delta\left(X_{1}(p)\right)$ orthogonal to $\mathbb{1}$ in $\mathcal{M}\left(X_{1}\right)$ is negative definite,

(ii). only if the restriction of the quadratic form $F_{2}(r, r)$ to measures orthogonal to $\mathbb{1}$ in $\mathcal{M}\left(X_{2}\right)$ is non-positive definite.

Proof The sufficient condition derives trivially from the proposition. The restriction to $X_{2}$ in the necessary condition insures that $p$ be in the relative interior of $\Delta\left(X_{2}\right)$, hence making it possible to violate the inequality in (3) if the quadratic form is not non-negative definite.

Concerning the nonlinear case, we need to introduce the following notation: ${ }^{6}$ define $H(x, p)=\mathrm{D}_{2} G(x, p)$ the derivative of $G$ with respect to $p$, and its restriction $\mathrm{H}_{2}$ to $X_{2} \times \Delta\left(X_{2}\right)$. It follows from the proof of theorem 3 below the following:

Theorem 2 a necessary condition for a Wardrop equilibrium $p$ to be an E.S.S. is that the restriction of the quadratic form $\left\langle r, H_{2}(p) r\right\rangle$ to $r \in \mathbb{1}^{\perp} \subset \mathcal{M}\left(X_{2}\right)$ be nonpositive definite.

Proof Introduce the (negative) score function ${ }^{7}$

$$
\mathcal{E}(\varepsilon, q)=F\left(q, q_{\varepsilon}\right)-F\left(p, q_{\varepsilon}\right),
$$

and notice that $B(p)=\{q \mid \mathcal{E}(0, q)=0\}$. Because $F$ is always linear w.r.t. its first argument, it follows that $\mathrm{D}_{1} \mathcal{E}(0, q)=\langle q-p, H(p)(q-p)\rangle$. The result follows as above.

\footnotetext{
${ }^{5}$ It would suffice that negativity be required of the quadratic form $\left\langle q_{1}-p_{1}, H_{1}\left(q_{1}-p_{1}\right)\right\rangle$ for all $q_{1} \in \Delta\left(X_{1}\right)$, which is less demanding. However, we offer no simple check of that property, it is why we sticked with the definition given here.

${ }^{6}$ There is a slight overload of notation for $H$ w.r.t. the linear case. It is resolved if we accept that $D_{2} G(x, q) r=H(x, q) r=\int_{X} H(x, y, q) r(\mathrm{~d} y)$.

${ }^{7}$ We borrow this name to [21]
} 
We may give a somewhat more explicit condition, but it is not clear that it be more useful. Let $\mu$ be the Lebesgue measure and $\mu_{1}=\mu\left(X_{1}\right)$. Given any measure $r$ over $X_{1}$, the measure $\hat{r}=r-\left(r\left(X_{1}\right) / \mu_{1}\right) \mu$ is orthogonal to $\mathbb{1}$. So that the first condition of our first theorem, e.g., reads

$$
\begin{aligned}
& \forall r \neq \alpha \mu \in \Delta\left(X_{1}\right) \text { for some } \alpha \in \mathbb{R}, \\
& F_{1}(r, r)-\frac{r\left(X_{1}\right)}{\mu_{1}}\left(F_{1}(r, \mu)+F_{1}(\mu, r)\right)+\frac{r\left(X_{1}\right)^{2}}{\mu_{1}^{2}} F_{1}(\mu, \mu)<0 .
\end{aligned}
$$

This is again a quadratic form over $\Delta\left(X_{1}\right)$. It could be translated in terms of $H$. This does not seem very useful. It is not so in the finite linear case.

\subsubsection{Finite linear case}

Assume now that $X=\left\{x_{1}, x_{2}, \ldots, x_{n}\right\}$ and that $G(p)$ is linear in $p$. We identify the function $H$ of (1) with the matrix with elements $H_{i j}=H\left(x_{i}, x_{j}\right)$. We now have $G(p)=H p$, and $F(q, p)=\langle q, H p\rangle=q^{t} H p$. (In general the marix $H$ is not symmetric.)

A distribution $p \in \Sigma_{n}$ is a Wardrop equilibrium if and only if, up to a reordering of the coordinates (hence of the $x_{i}$ ), it can be written as a composite vector

$$
p=\left(\begin{array}{c}
p_{1} \\
0
\end{array}\right)
$$

with $p_{1} \in \Sigma_{n_{1}} \subset \mathbb{R}^{n_{1}}$, with furthermore

$$
H\left(\begin{array}{c}
p_{1} \\
0
\end{array}\right)=\left(\begin{array}{c}
f \mathbb{1} \\
z
\end{array}\right)
$$

for the same decomposition, with $f \in \mathbb{R}$ and $z_{j}<f$ for every coordinate $z_{j}$ of $z$. Here, $Z(p)$ is the set $\left\{x_{n_{1}+1}, \ldots, x_{n}\right\}, B(p)$ is the set of all $q \in \Sigma_{n}$ which share the same decomposition $\left(q_{1}, 0\right)$ as $p$, and $F(p, p)=f$.

To investigate the second order condition, we partition $H$ according to the same decomposition in

$$
H=\left(\begin{array}{ll}
H_{11} & H_{10} \\
H_{01} & H_{00}
\end{array}\right),
$$

and we identify $H_{11}$ with the restriction $H_{1}$ of $H$ to $X_{1} \times X_{1}$.

The sufficient condition of theorem 1 is now that the restriction of the quadratic form $\left\langle r_{1}, H_{1} r_{1}\right\rangle$ to vectors $r_{1}$ orthogonal to the vector $\mathbb{1}$ of $\mathbb{R}^{n_{1}}$ be negative definite. Furthermore, call $p_{2}$ the sub-vector of the strictly positive coordinates of $p_{1}$. The necessary condition of theorem 1 is that the corresponding sub-matrix $H_{2}$ generate a non-positive definite restriction of the quadratic form $\left\langle r_{2}, H_{2} r_{2}\right\rangle$ to vectors $r_{2}$ orthogonal to $\mathbb{1}$ (with appropriate dimension). 
We now give a practical means of checking these conditions. Let first $A$ be a $2 \times 2$ matrix

$$
A=\left(\begin{array}{ll}
a & b \\
c & d
\end{array}\right) .
$$

We note $\sigma(A)$ the symmetric difference of the four numbers:

$$
\sigma(A)=a-b-c+d .
$$

Then, given a $m \times m$ matrix $A$, define $\sigma(A)$ as the $m-1 \times m-1$ matrix obtained by replacing each block of four adjacent entries in $A$ by their symmetric difference.

Lemma 1 The resriction of the quadratic form $\langle r, A r\rangle$ to the vectors $r$ orthogonal to $\mathbb{1}$ is negative definite (resp. non-positive definite) if and only if $\sigma(A)+\sigma(A)^{t}$ is negative definite (resp. non-positive definite).

Proof $\sigma(A)=P^{t} A P$ where $P$ is the $m \times m-1$ injective matrix

$$
P=\left(\begin{array}{cccc}
1 & 0 & \cdots & 0 \\
-1 & 1 & \cdots & 0 \\
0 & -1 & \cdots & 0 \\
\vdots & \vdots & \ddots & \vdots \\
0 & 0 & \cdots & 1 \\
0 & 0 & \cdots & -1
\end{array}\right)
$$

whose range space is the vector sub-space orthogonal to the vector $\mathbb{1}$.

\subsection{Further stability concepts}

Many variations of the concept of E.S.S. have been proposed. The only one we mention here is sometimes called Evolutionary Robust Strategy or E.R.S. ${ }^{8}$ It is as follows:

Definition 3 A strategy distribution $p$ is called an E.R.S. if there exists a weak neighborhood $\mathcal{N}$ of p such that

$$
\forall q \neq p \in \mathcal{N}, \quad F(p, q)>F(q, q) .
$$

Hence, in effect, for an E.R.S., the dominance inequality of (6) is requested of all $q$ in a neighborhood and not only of the best response $q$ 's.

Concerning the relationship of this concept with E.S.S., we recall the notation $H(x, p)=\mathrm{D}_{2} G(x, p)$ the derivative of $G$ with respect to $p$, and its restrictions $H_{1}$ to $X_{1} \times \mathcal{M}\left(X_{1}\right)$ and $H_{2}$ to $X_{2} \times \mathcal{M}\left(X_{2}\right)$. We state:

\footnotetext{
${ }^{8}$ It is closely related to J. Apaloo's concept of Neighborhood Invader Strategies [2]
} 
Definition 4 A Wardrop equilibrium $p$ is said regular if there exists a $\Delta\left(X_{1}\right)$ neighborhood of $p$ such that over that neighborhood $q \mapsto H_{1}(x, q)$ is Lipshitz continuous for all $x \in X^{9}$ and the restriction of the quadrtic form $\left\langle r, H_{1}(q) r\right\rangle$ to measures $r$ orthogonal to $\mathbb{1}$ in $\mathcal{M}\left(X_{1}\right)$ is negative definite, both uniformly in $q$.

we have the following

\section{Theorem 3}

(i). All E.R.S. are E.S.S.

(ii). In the finite case, all regular Wardrop equilibria, and all E.S.S. in the linear case, are E.R.S.

The proof of $(i)$ is elementary: applying the E.R.S. condition to $q_{\varepsilon}=p+\varepsilon(q-p)$ and using the linearity of $F$ w.r.t. its first argument, we get condion (3). The proof of (ii) is classical for the finite linear case (see [8]). We extend it to the nonlinear regular case in the appendix.

The importance of the concept of E.R.S. for us lies in the fact that it is a (local) Lyapunov asymptotically stable point of the replicator dynamics.

\subsection{Clutch size determination and Braess'paradox}

The transportation literature is familiar with Braess'paradox, seldom quoted in the E.S.S., biologically-inspired, literature. This generically refers to situations where improving the quality of the resource decreases the fitness of every individuals in the population. The following is such an example.

A species of paraitoids lays its eggs in its hosts, say the eggs of another species. This is a gregarious parasitoid, meaning that several offspring can be born from a single host. Yet, the probability that the parasitizing succeed, actually giving parasitoid newborns, decreases with the number of eggs in the host. Eiher all eggs layed in a any host succeed or all fail. The population of parasitoids is such that, as a first approximation, it may be assumed that every host will be superparasitized once, meaning that exactly two parasitoids will lay some eggs in each host. We are interested in the number of eggs, or clutch size, $x$ that the female parasitoids lay.

We consider a very simple case where only three parasitoids can be born from a single host. We assume that if only two parasitoid eggs are laid in a single host, they will survive (with probability one). If three are laid, the survival probability is $\pi$, and we assume $\pi>1 / 3$. The only two sensible (pure) stategies are laying one or

\footnotetext{
${ }^{9}$ We shall use this definition in the finite case only, it is why we need not assume any regularity w.r.t. $x$.
} 
two eggs, since at least one more will be present in the host due to superparasitism. The game matrix of this problem is as follows:

$$
G=\left(\begin{array}{cc}
1 & \pi \\
2 \pi & 0
\end{array}\right)
$$

The Wardrop equilibrium is $p(\{x=1\})=\pi /(3 \pi-1)$. It is an E.S.S., since $\sigma(G)=1-3 \pi<0$. The collective fitness at equilibrium is

$$
F(p, p)=\frac{2 \pi^{2}}{3 \pi-1} .
$$

For $\pi=1 / 2$, this leads to $F(p, p)=1$, while improving the survival probability of a group of three eggs to $\pi=2 / 3$, we obtain $p=2 / 3$ and $F(p, p)=8 / 9<1$.

\section{Replicator dynamics}

We shall consider only the finite case. The infinite case is far less well known. Some results concerning it, both old an new, can be found in [15].

\subsection{Evolutionary dynamics}

Assume that "fitness" $G_{i}(p)$ measures the excess of the number of birth over the number of death (may be negative) per individuals using the strategy $x_{i}$ (with phenotype $x_{i}$ ) in a population of strategy distribution $p$. Let $n_{i}(t)$ be the number of individuals using $x_{i}$ at time $t$. We also have for the strategy distribution

$$
q_{i}(t)=\frac{n_{i}(t)}{\sum_{k} n_{k}(t)}
$$

If generations are discrete, with a time step $h$, — say a population reproducing once a year- this yields

$$
n_{i}(t+h)=\left[1+h G_{i}(q(t))\right] n_{i}(t),
$$

which, together with the definition of $q_{i}(t)$ gives ${ }^{10}$

$$
q_{i}(t+h)=q_{i}(t) \frac{1+h G_{i}(q(t))}{1+h F(q(t), q(t))} .
$$

\footnotetext{
${ }^{10}$ The the classical theory summarized here departs from the discrete time dynamics of [10]
} 
Looking at these dynamics over a very large time horizon, i.e. taking a larger and larger time unit, is equivalent to letting $h$ go to zero. And the above equation converges to the replicator dynamics

$$
\dot{q}_{i}=q_{i}\left[G_{i}(q)-F(q, q)\right]
$$

We summarize here the classical invariance and asymptotic properties of these equations:

\section{Theorem 4}

(i). They leave each face of $\Sigma_{n}$ invariant,

(ii). All limit points of trajectories of the replicator dynamics are Wardrop equilibria.

(iii). In finite dimension, all E.R.S. are locally asymptotically stable.

\section{Proof}

(i). Any $q_{i}(0)=0$ is preserved. And the defect $D(q):=1-\sum_{i} q_{i}$ satisfies $\dot{D}(q)=-D(q) F(q, q)$ and therefore leaves 0 invariant.

(ii). If $p$ is not Wardrop, for some $i, G_{i}(p)-F(p, p)>0$, and by continuiy, $G_{i}(q)-F(q, q)>0$ for $q$ in a neighborhood of $p$. Noticing that

$$
q_{i}(t)=q_{i}\left(t_{0}\right) \exp \left(\int_{t_{0}}^{t}\left[G_{i}(q(s))-F(q(s), q(s))\right] \mathrm{d} s\right),
$$

we have a clear contradiction with convergence to $p$.

(iii). Let $p$ be en E.R.S. Use as a Lyapunov function the relative entropy of $q$ with respect to $p$ :

$$
V(q)=\sum_{i \in X_{1}} p_{i} \ln \left(\frac{p_{i}}{q_{i}}\right) .
$$

It is straightforward to check that it is decreasing along any trajectory, and continuous with respect to any topology on $\Delta(X)$ - and is therefore a valid Lyapunov function, which does not hold in infinite dimension.

This equation has been extensively used as a simple model of population dynamics under evolution [10, 21, 8] and also as a Nash equilibrium selection device [13]. 


\subsection{Population games}

\subsubsection{Population game dynamics}

The ideas embedded into the derivation of the replicator dynamics may be extended to situations where several different populations are interfering, in effect, to Nash equilibria of classical games as opposed to Wardrop equilibria. These have been called "Population games" by W. Sandholm [14].

Let $N$ populations interact. We denote the population number with upper indices $K, L=1,2, \ldots, N$. Individuals of population $K$ have $n^{K}$ possible strategies (phenotypes). As previously, we let $n_{i}^{K}$ be the number of individuals of population $K$ using the strategy $i, q_{i}^{K}$ be the proportion of such individuals in population $K$, and $q^{K}$ be the $n^{K}$-dimensional vector of the $q_{i}^{K}, i=1,2, \ldots, n^{K}$. We shall also make (parcimonious) use of the classical notation $q^{-K}$ to mean the set of all $q^{L}$ for $L \neq K$, and let $q$ be the set of all $q^{K}$ 's. It is assumed that the fitness of an individual of population $K$ using strategy $i$ is a function $G_{i}^{K}\left(q^{-K}\right)$, so that the collective fitness of population $K$ is $F^{K}(q)=F^{K}\left(q^{K}, q^{-K}\right)=\left\langle q^{K}, G^{K}\left(q^{-K}\right)\right\rangle$.

W. Sandholm has investigated several mechanisms by which the individual may update their choices of strategies. Several of the most natural schemes lead to the dynamics

$$
\dot{n}_{i}^{K}=n_{i}^{K} G_{i}^{K}\left(q^{-K}\right) .
$$

This yields

$$
\dot{q}_{i}^{K}=q_{i}^{K}\left[G_{i}^{K}\left(q^{K}\right)-F^{K}(q)\right] .
$$

\subsubsection{Wolves and Lynxes}

We restrict now our attention to linear two-population games with two strategies available in each population. This is the simplest possible case, and it already exhibits interesting features.

Let therefore $G^{1}$ and $G^{2}$ be two $2 \times 2$ matrices,

$$
G^{K}=\left(\begin{array}{ll}
a^{K} & b^{K} \\
c^{K} & d^{K}
\end{array}\right)
$$

\begin{tabular}{|c|c|c|}
\hline \multicolumn{2}{|c|}{$x^{1} \backslash x^{2}$} & 2 \\
\hline & $a$ & $c$ \\
\hline 1 & $a^{1}$ & $b^{1}$ \\
\hline & $b$ & $d^{2}$ \\
\hline 2 & $c^{1}$ & $d^{1}$ \\
\hline
\end{tabular}

so that the payoffs are $G^{K}\left(q^{L}\right)=G^{K} q^{L}$ with transparent abuses of notations. The underlying two-player game is defined by the bi-matrix 
Furthermore, the vectors $q^{K}, K=1,2$, will be represented by their first component, i.e. again with a transparent abuse of notation

$$
q^{K}=\left(\begin{array}{c}
q^{K} \\
1-q^{K}
\end{array}\right)
$$

We let $\sigma^{K}$ be the symmetric difference $a^{K}-b^{K}-c^{K}+d^{K}$, and whenever $\sigma^{L} \neq 0$, define $p^{K}=\left(d^{L}-b^{L}\right) / \sigma^{L}, L \neq K$.

We are here interested in the case where both $p^{K}$ exist and lie in $(0,1)$. They constitute a mixed Nash equilibrium. It is a simle matter to see that if both $\sigma^{K}$ have the same sign, there are in addition two pure nash equilibria, diagonally opposite in the square $[0,1] \times[0,1]$, while if they are of opposite sign, this is the only Nash equilibrium.

The replicator dynamics read

$$
\dot{q}^{K}=\sigma^{L} q^{K}\left(1-q^{K}\right)\left(q^{L}-p^{L}\right), \quad L=3-K .
$$

Their behavior is characterized in part by the following result (see $[8,4]$ for a more detailed analysis)

Theorem 5 If $\sigma^{1} \sigma^{2}<0$, the point $\left(p^{1}, p^{2}\right)$ is a center of the replicator dynamics, and all trajectories are periodical. If $\sigma^{1} \sigma^{2}>0$, the point $\left(p^{1}, p^{2}\right)$ is a saddle, and the two pure Nash equilibria are asymptotically stable.

Proof Consider the relative entropies:

$$
U^{K}\left(q^{K}\right)=p^{K} \ln \frac{p^{K}}{q^{K}}+\left(1-p^{K}\right) \ln \frac{1-p^{K}}{1-q^{K}}
$$

and the function

$$
V\left(q^{1}, q^{2}\right)=\sigma^{2} U^{1}\left(q^{1}\right)-\sigma^{1} U^{2}\left(q^{2}\right) .
$$

A straightforward computation shows that its Lagrangian derivative is null. The $U^{i}$ 's are convex, and go to infinity as $p^{K}$ approaches 0 or 1 . If $\sigma^{1} \sigma^{2}<0, V$ is either convex or concave, diverging to plus or minus infinity as $\left(q^{1}, q^{2}\right)$ approaches the boundary of the domain of definition. The tajectories are level curves of $V$, which in that case are the boundaries of convex level sets contained in the domain. If $\sigma^{1} \sigma^{2}>0$, the curve $V\left(q^{1}, q^{2}\right)=0$ separates the attraction basins of the two pure Nash equilibria.

We provide an example in a "hawk and doves" type of games, but between two populations sharing the same preys, say wolves and lynxes hunting deer, but where contests occur only between individual of different species. Each have two possible behaviours, $H$ for "hawkish" and $D$ for "Dovish". 
In that model, Lynxes are at a trophic level above that of wolves. In particular, if two agressive individuals meet, the lynx is hurt, but the wolf is killed. We also assume that against a pacific (coward) wolf, an agressive lynx gets less than 1 (the full benefit of the prey), because it has spent unnecessary time and effort chasing a competitor who would have left anyhow.

The result is the following bi-matrix of rewards:

\begin{tabular}{c|cc|cc|}
\multicolumn{1}{l}{$L \backslash W$} & $D$ & & $H$ \\
\cline { 2 - 5 }$D$ & $\lambda$ & $1-\lambda$ & & 1 \\
$D$ & $\lambda$ & & 0 & \\
\hline & & 0 & \multicolumn{2}{|c}{$-\theta$} \\
$H$ & $1-\mu$ & & $1-\nu$ & \\
\hline
\end{tabular}

with $\lambda+\mu>1>\nu>0$ and $\theta>0$. In that game, we have $\sigma_{1}=\lambda+\mu-\nu$ and $\sigma_{2}=-\lambda-\theta$, and the Nash strategies $p^{1}=\theta /(\lambda+\theta), p^{2}=(1-\nu) /(\lambda+\mu-\nu)$. Figure 1 shows a typical trajectory, computed in the case $\lambda=\nu=1 / 2, \theta=2 \mu=$ 1.5 , and intial state $(0.2,0.2)$.

\subsubsection{Joint interest and bifurcation}

Maynard-Smith [10] considers the case where both populations share a joint foe, say Man. Then, there is a benefit for each population in maintaining the other one, as it contributes to keep that foe away. This is in effect an equivalent in population games of the concept of inclusive fitness in E.S.S. See [7] and the references there in. We address a side-topic on these lines, staying with our simple Wolves and Lynxes game.

Several ways of taking into account that joint interest have been proposed in the literature, depending on the detailed mechanisms at work. One possibility is by saying that each population $K$ has a fitness $F_{\alpha}^{K}(q)=(1-\alpha) F^{K}(q)+\alpha F^{L}(q)$ for some coefficient $\alpha \in[0,1 / 2]$. Then, we get

$$
F_{\alpha}^{K}(q)=(1-\alpha)\left\langle q^{K}, G^{K} q^{L}\right\rangle+\alpha\left\langle q^{L}, G^{L} q^{K}\right\rangle=\left\langle q^{K}, G_{\alpha}^{K} q^{L}\right\rangle
$$

with $G_{\alpha}^{K}=(1-\alpha) G^{K}+\alpha\left(G^{L}\right)^{t}$.

Now, the behaviour of the replicator equation obviously depends on the coefficient $\alpha$ measuring the amount of joint interest. As an example, we work out the Wolves and Lynxes problem with $\lambda=\nu=1 / 2, \theta=2 \mu=3 / 2$. For $\alpha<1 / 6$, the dynamics are qualitatively similar to the case of figure 1: we have periodical trajectories revolving clockwise around the mixed Nash equilibrium $\left(p^{1}, p^{2}\right)$. As $\alpha$ increases, $p^{2}$ decreases, so that the band of trajectories going leftward at the bottom of the square $[0,1] \times[0,1]$ shrinks. Simultaneously, the speed of the dynamics 


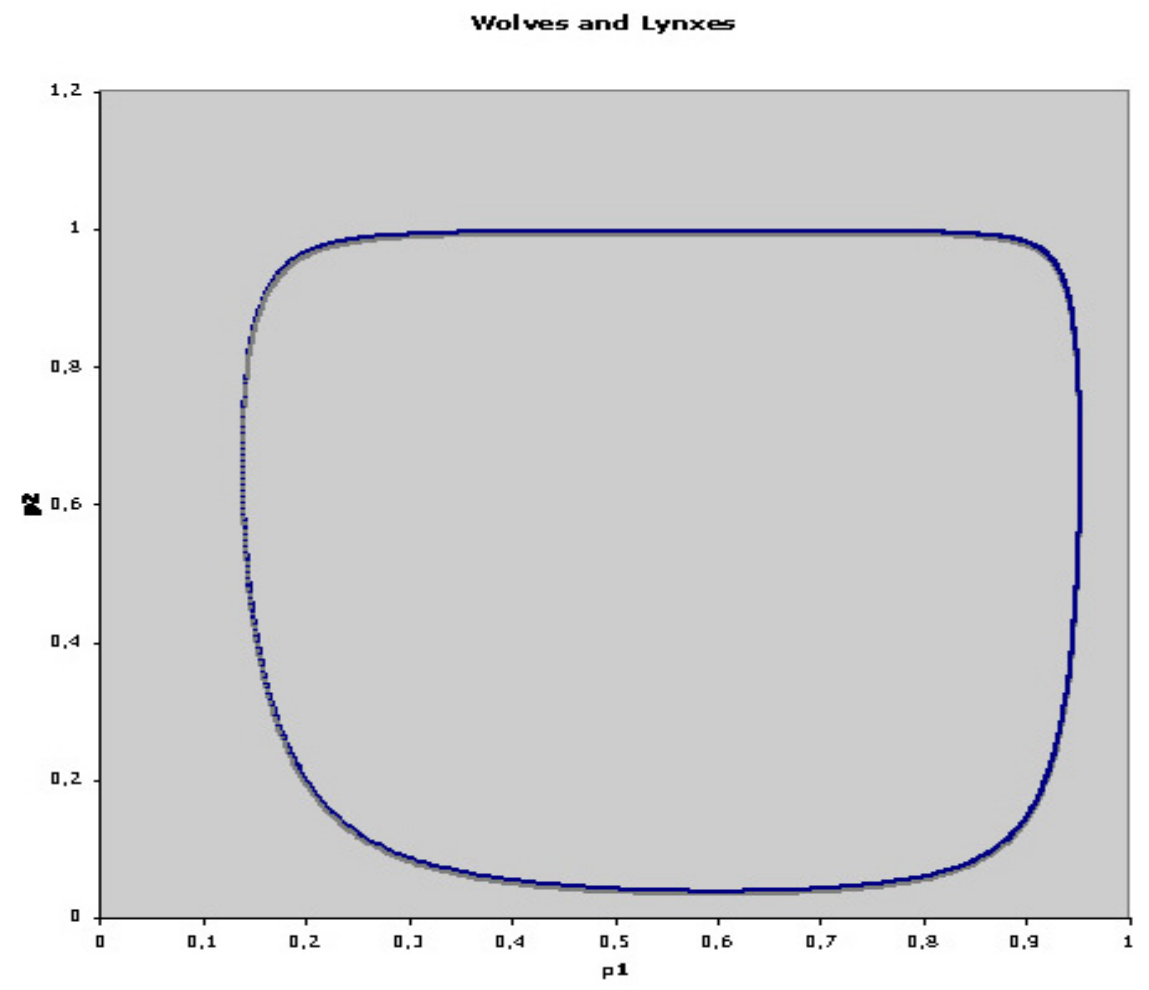

Figure 1: Population dynamics for Wolves and Lynxes, $\lambda=\nu=1 / 2, \theta=2 \mu=$ 1.5 , time span : 40 units.

to the left decreases. At $\alpha=1 / 6, p^{2}$ reaches 0 . At that precise value, the lower trajectories going left have disappeared, giving way to a line of rest points on the $q^{1}$ axis. These equilibria are unstable for $q^{1}<p^{1}=29 / 37$, with trajectories leaving "upward" from them, and stable (or at least the line of equilibria is stable) for $q^{1}>29 / 37$.

For larger values of $\alpha$ up to $1 / 2$, the only Nash equilibrium is $(1,0)$. All trajectories of the replicator dynamics converge to that point, including the trajectory $q^{2}=0$ where the velocity has been reversed and now points to the right. This is pictured in figure ??

The conclusion is that a small variation in the parameter $r$ may cause a drammatic difference in the qualitative behaviour of the dynamics. That parameter is, at best, difficult to estimate. But a reverse use of this theory can be made, deriving from the observed behaviour bounds for possible values of $\alpha$ if this model is to be used. 

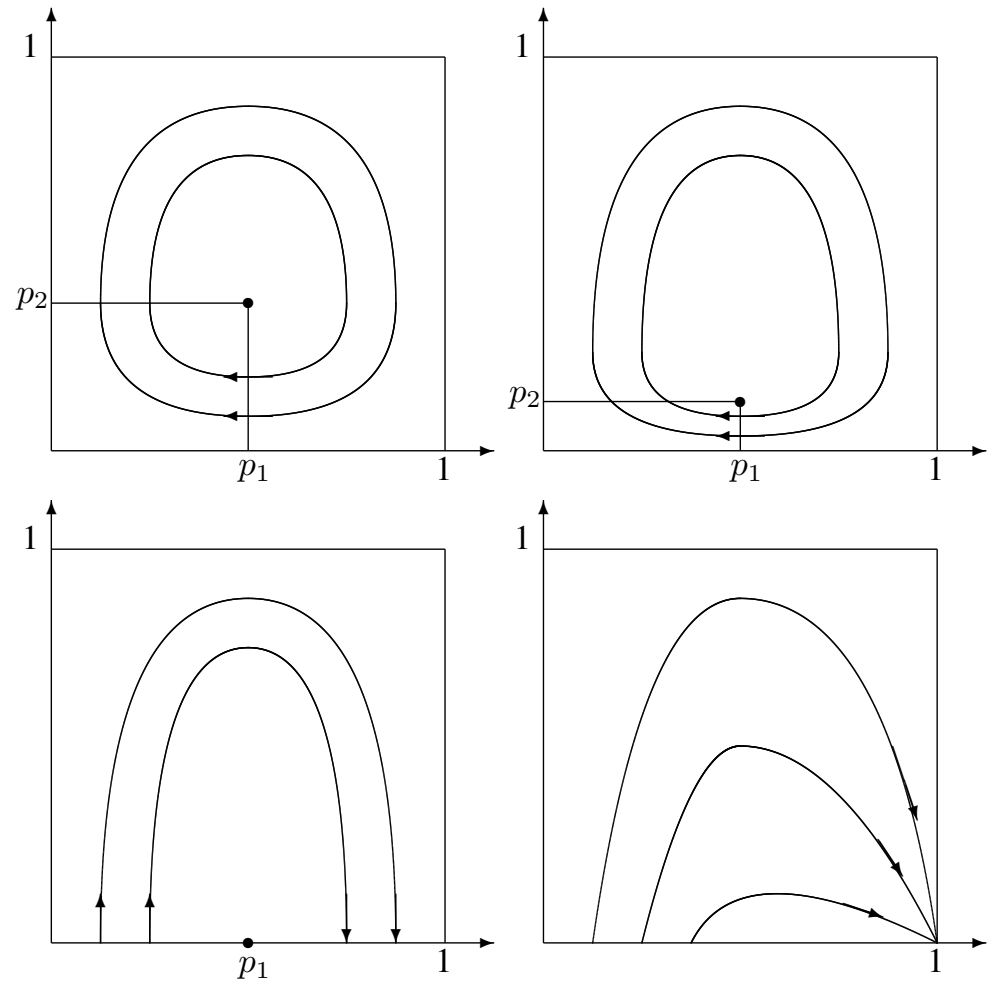

Figure 2: The bifurcation as the joint interest raises, left to right and top to bottom, from 0 to $1 / 2$

Notice that in the last case, $\alpha$ large, there is a single Nash equilibrum at $(1,0)$. We are no longer in the case where $p_{2} \in(0,1)$ where pure Nash equilibria came in pairs.

\section{Dynamic generating function}

We now venture with two examples in the realm of Wardrop equilibria with infinite dimensional trait spaces. Many examples can be found in the literature, see e.g. [7]. These are two different examples which lead to tractable Wardrop equilibria computations. 


\subsection{The tragedy of the commons}

In this example, remotely inspired by [12], individuals of a population may use two strategies, say $x \in\{0,1\}$. However, they have to make that choice at each instant of time $t \in[0, T]$ over a fixed horizon $T$, say the breeding season. So that the strategy set is the set of (measurable) functions $x(\cdot):[0, T] \rightarrow\{0,1\}$. As usual, we shall let $q(t)$ be the proportion of individuals in the population using $x=1$ at time $t$, and denote $p(\cdot)$ a Wardrop equilibrium.

Their collective behaviour has an influence on the "state of the world", say environmental quantities such as amount of resources that they may be depleting, habitat quality, etc. This is modelized as a vector dynamical system in $\mathbb{R}^{m}$ :

$$
\dot{y}=f(y, q), \quad y(0)=y_{0},
$$

where $f$ is assumed of class $C^{1}$, and is such that this differential equation has a solution over $[0, T]$ for all measurable $q(\cdot)(q(t) \in[0,1])$.

The time rate of fitness acquisition by an individual using $x=1$ is a scalar function $g(y)$, also of class $C^{1}$, while individuals using $x=0$ gather no fitness. ${ }^{11}$ As a result, the cumulative fitness at the end of the season is

$$
G(x(\cdot), q(\cdot))=\int_{0}^{T} x(t) g(y(t)) \mathrm{d} t .
$$

To ease the calculations, we let

$$
A(y, q):=\mathrm{D}_{1} f(y, q)=\frac{\partial f}{\partial y}, \quad b(y, q):=\mathrm{D}_{2} f(y, q)=\frac{\partial f}{\partial q} .
$$

Having a resource depletion problem in mind, we assume that

- If all individuals use the available resources, the quality of the environment, as measured by $g(y)$, decreases, and if none does, the environment regenerates, and $g$ increases:

$$
\mathrm{D} g(y) f(y, 1)<0, \quad \mathrm{D} g(y) f(y, 0)>0,
$$

- for all $y$ such that $g(y)=0$,

$$
\mathrm{D} g(y) b(y, q)<0 .
$$

\footnotetext{
${ }^{11}$ Or $g$ is the increase of fitness provided by $x=1$.
} 
As long as $g(y(t))>0$, the clear optimum for every individuals is to use $x=1$, so that a Wardrop equilibrium, and a fortiori an E.S.S., must have $q(t)=1$. Conversely, as long as $g(y(t))<0$, we end up with $q(t)=0$. The only possible mixed Wardrop equilibrium is therefore with $g(y)=0$, hence at pairs $(y(\cdot), q(\cdot))$ satisfying

$$
\mathrm{D} g(y) f(y, q)=0
$$

Thanks to hypotheses (10) and (11), equation (12) defines a unique smooth implicit function $q=\varphi_{0}(y) \in(0,1)$. Altogether, this defines a closed loop Wardrop equilibrium $p(t)=\varphi(y(t))$ with

$$
\varphi(y)= \begin{cases}1 & \text { if } g(y)>0 \\ \varphi_{0}(y) & \text { if } g(y)=0 \\ 0 & \text { if } g(y)<0\end{cases}
$$

This will cause the $y$ trajectory to reach $\{g(y)=0\}$ at a time $t_{0}$ with $p=1$ if $g\left(y_{0}\right)>0$, and with $p=0$ if $g\left(y_{0}\right)<0$, and then to follow a path in the manifold $g(y)=0$. We denote by $z(\cdot)$ that trajectory. The ensuing collective fitness is

$$
F(p, p)=\int_{0}^{t_{0}}[g(z(t))]^{+} \mathrm{d} t .
$$

We now investigate the second order condition to check whether that Wardrop equilibrium is also an E.S.S. Since using a mixed strategy while $g(y) \neq 0$ is clearly non optimal, we shall only consider the time interval $\left[t_{0}, T\right]$ during which $g(z(t))=0$. In order to apply theorem 2, introduce the transition matrix $\Phi(t, s)$ of the matrix $A(z(t), p(t))$. We have

$$
\left\langle r, H_{2}(p) r\right\rangle=\iint_{\mathcal{T}} r(t) \mathrm{D} g(z(t)) \Phi(t, s) b(z(s), p(s)) r(s) \mathrm{d} s \mathrm{~d} t,
$$

where $\mathcal{T}$ is the triangle $t_{0} \leq s \leq t \leq T$. Let therefore the scalar function $h(\cdot, \cdot)$ be defined as

$$
h(t, s)=\mathrm{D} g(z(t)) \Phi(t, s) b(z(s), p(s)),
$$

the second order E.S.S. condition reads

$$
\iint_{\mathcal{T}}(q(t)-p(t)) h(t, s)(q(s)-p(s)) \mathrm{d} s \mathrm{~d} t<0 .
$$

Theorem 6 A necessary condition for the Wardrop equilibrium described above to be an E.S.S. is that, for any pair $s<t \in\left[t_{0}, T\right], h(t, t)-2 h(t, s)+h(s, s) \leq 0$. 
Proof Select a small time interval $\eta$, and $\alpha \neq 0$ such that, for $\tau \in(s, s+\eta)$, $p(\tau)+\alpha \in(0,1)$ and for $\tau \in(t-\eta, t), p(\tau)-\alpha \in(0,1)$. Choose

$$
q(\tau)= \begin{cases}p(\tau)-\alpha & \text { for } \tau \in[s, s+\eta] \\ p(\tau)+\alpha & \text { for } \tau \in[t-\eta, t] \\ p(\tau) & \text { otherwise }\end{cases}
$$

Placing this $q$ in (13), we see that $(2 / \alpha \eta)\left\langle p-q, H_{2}(p-q)\right\rangle \rightarrow h(t, t)-2 h(t, s)+$ $h(s, s)$ as $\eta$ goes to zero.

We leave it to the reader to check the conjecture that a sufficient condition is that $h(t, t)-2 h(t, s)+h(s, s)<0$ for all $s<t \in\left[t_{0}, T\right]$.

A simple example is the following dynamic form of the famous "Tragedy of the Commons" game, of which we provide two variants. Here $y$ is scalar and measures the amount of the common resource available, say the pasture shared by shepherds.

In the first variant, we use a simple malthusian dynamics:

$$
\dot{y}=a y+b q+c
$$

with $a>0$, a rate of natural regeneration, $b<0$, the depletion rate if all the local flocks graze in that same common pasture, and $c>0$, a zero-resource regeneration rate. $^{12}$ The efficiency of sheep grazing is $g(y)=y-\gamma$ for some fixed level $\gamma<-(b+c) / a$. The equilibrium state is $z=\gamma$, and the equilibrium strategy distribution $p=(a \gamma+c) /(-b)$. We get $h(t, s)=b \exp [a(t-s)]$. Hence, we find that $h(t, t)-2 h(t, s)+h(s, s)=-2 b(\exp [a(t-s)]-1)>0$ failing the necessary condition. Therefore the Wardrop equilibrium is not an E.S.S.

In a second variant, we replace the linear growth by a more realistic logistic growth:

$$
\dot{y}=\alpha\left(1-\frac{y}{K}\right) y+b q+c,
$$

with $b<-\alpha K / 4$, leaving $g$ unchanged. Now, $A(z, p)=\alpha(1-2 \gamma / K)=a$. If $\gamma \in(K / 2, K), a<0$ and the conclusion is reversed : we have indeed an E.S.S. If $\gamma$ is thought of as a tax for using the common resource, this may provide a hint at how to choose it.

We do not know the full implication of these comparative results, and in particular whether the replicator equation converges to the equilibrium in the second case, say.

\footnotetext{
${ }^{12}$ We need to restrict $y(0)$ to be less than $-(b+c) / a$ to insure hypothesis (10).
} 


\subsection{Routing in a dense network}

We consider now a problem close to transportation theory, where the Wardrop equilibrium originated, posed by Eitan Altmann and investigated in more detail in [1].

There is given an open region $\Omega$ of the plane with a smooth boundary $\Gamma$. We shall let $y \in \mathbb{R}^{2}$ represent the coordinates of a point in $\Omega$. At each $y \in \Gamma$ we denote $n(y)$ the outward normal. An ad-hoc communication network covers $\Omega$. The routers are so numerous that we modelize the situation as a continuum of routers. The boundary $\Gamma$ of $\Omega$ is divided into two disjoint parts: $\Gamma=\mathcal{Q} \cup \mathcal{R}$. On $\mathcal{Q}$, a density $\sigma(y) \geq 0$ of messages enter $\Omega$. Part of $\mathcal{Q}$ may have $\sigma(y)=0$, meaning that no message should either enter or leave through that part. A density $\rho(y) \geq 0$ of messages is generated at each point $y \in \Omega$. All messages must leave through $\mathcal{R}$.

A strategy is the choice of a vector field $x(\cdot): \Omega \rightarrow \mathbb{R}^{2}$ representing the flow of messsages at each point. The intensity of the flow is $\|x\|$. To be admissible, such a vector field must satisfy

$$
\begin{array}{ll}
\forall y \in \mathcal{Q}, & \langle n(y), x(y)\rangle=-\sigma(y), \\
\forall y \in \Omega, & \operatorname{div} x(y)=\rho(y) .
\end{array}
$$

There is a congestion cost in terms of transmission delay. The average time to transmit one message by the router $y$ is $\tau(y)$, so that the delay encountered by one message going through the router $y$ is $\tau(y)\|x(y)\|$.

We consider a lone message whose path does not change the overall field. It is generated at $y_{0}$ which may belong to $\Omega \cup \mathcal{Q}$, follows a path $y(s), s$ its curvilinear abscissa along the path, and leaves $\Omega$ at $y_{1} \in \mathcal{R}$. Let $f$ be the unit vector giving its direction of travel. The total time of travel will be given by the line integral

$$
G=\int_{y_{0}}^{y_{1}} \tau(y)\|x(y)\| \mathrm{d} s
$$

with

$$
\frac{\mathrm{d} y(s)}{\mathrm{d} s}=f(s) .
$$

A field of messages flow will be considered a Wardrop equilibrium if given the congestion it produces, the fastest path for any message originating in $\Omega \cup \mathcal{Q}$ is to follow that field.

We investigate that last optimization problem via its Hamilton Jacobi Bellman equation. Let $V\left(y_{0}\right)$ be the minimum time from $y_{0}$ to $\mathcal{R}$. It is a viscosity solution of the P.D.E.

$$
\begin{aligned}
& \forall y \in \Omega, \quad \min _{\|f\|=1}\langle f, \nabla V(y)\rangle+\tau(y)\|x(y)\|=0, \\
& \forall y \in \mathcal{R}, \quad V(y)=0 .
\end{aligned}
$$


The obvious optimum is $f=-\nabla V /\|\nabla V\|$ and $\|\nabla V(y)\|=\tau(y)\|x(y)\|$.

Therefore, $x(\cdot)$ is a Wardrop equilibrium if

$$
x(y)=-(1 / \tau(y)) \nabla V(y)
$$

Using furthermore the admissibility conditions (14), it comes

$$
\begin{array}{ll}
\forall y \in \mathcal{Q}, & \langle n(y), \nabla V(y)\rangle=-\sigma(y) \tau(y), \\
\forall y \in \mathcal{R}, & V(y)=0, \\
\forall y \in \Omega, & \operatorname{div}\left(\frac{1}{\tau(y)} \nabla V(y)\right)=\rho(y) .
\end{array}
$$

This is a classical elliptic problem with mixed Neumann-Dirichlet boundary conditions. For any particular case, it can be efficiently solved numerically. It provides the Wardrop equilibrium field via equation (15). As long as $\nabla V$ is nowhere zero in $\Omega$, which will always be the case if e.g. $\rho$ itself is everywhere positive, it is a pure strict Wardrop equilibrium, and thus an E.S.S.

\section{Conclusion}

We have opened this leisurely walk through Wardrop equilibria, E.S.S. and replicator equations by Wardrop's invention in road engineering and ended it by an example in a modern routing problem. This is as a tribute to its origins, and to stress the many domains where these concepts are used. But theoretical biology is the field that has brought more research into evolutionary games lately and made it a full fledged theory in itself.

As far as we know, the question of convergence of the replicator equations towards an E.R.S. in the infinite case is not settled at this time. This is only one of the open questions left. But our feeling is that many interesting problems lie in particular applications, whether finite or infinite, possibly even with infinite dimensional strategy space as in the last two examples we provided.

\section{References}

[1] E. Altman, P. Bernhard, M. Debbah, And A. Silva, The mathematics of routing in massively dense ad-hoc networks, in AdHoc-NOW 2008, Sophia Antipolis, 2008.

[2] J. Apaloo, Revisiting matrix games: The concept of neighborhood invader strategies, Theoretical Population Biology, 69 (2006), pp. 235-242. 
[3] M. Beckman, A continuous model of transportation, Econometrica, 20 (1952), pp. 643-660.

[4] P. Bernhard And F. Hamelin, Two-by-two static, evolutionary, and dynamic games, in From Semantics to Computer Science: Essays in Honor of Gilles Kahn, Y. Bertot, G. Huet, J.-J. Lévy, and G. Plotkin, eds., Cambridge University Press, 2008.

[5] D. T. Bishop And C. CANnings, A generalized war of attrition, Journal of Theoretical Biology, 70 (1978), pp. 85-125.

[6] R. CRessman, Evolutionary Dynamics and Games in Extensive form, M.I.T. Press, 2003.

[7] T. DAY AND P. D. TAYLOR, A generalization of pontryagin's maximum principle for dynamic evolutionary games among relatives, Theoretical Population Biology, 57 (2000), pp. 339-356.

[8] J. Hofbauer And K. Sigmund, Evolutionary Games and Population Dynamics, Cambridge University Press, Cambridge, U.K., 1998.

[9] J. MAYNARD SMITH, The theory of games and the evolution of animal conflicts, Journal of Theoretical Biology, 47 (1974), pp. 209-221.

[10] — Evolution and the Theory of Games, Cambridge University Press, Cambridge, U.K., 1982.

[11] J. Maynard Smith And G. R. Price, The logic of animal conflict, Nature, 246 (1973), pp. 15-18.

[12] B. Romagny, C. Lobry, And M. CAnAlis-Durand, Tragedy of the commons and prisoner's dilemma, Tech. Rep. 97A20, GREQAM, 1997.

[13] L. SAmuelson, Evolutionary Games and Equilibrium Selection, MIT Press, Cambridge, Massachusetts, USA, 1997.

[14] W. SAndholm, Population Games, M.I.T. Press, To appear.

[15] A. Shaiju AND P. BERnhARD, Evolutionarily stable strategies : Two nontrivial examples and a theorem, in International Symposium on Dynamic Games and Applications, no. 12, Sophia Antipolis, France, 2006.

[16] P. TAYLOR AND L. JONKER, Evolutionarily stable strategies and game dynamics, Mathematical Bioscience, 40 (1978), pp. 145-156. 
[17] T. L. Vincent And J. S. Brown, Evolutionary Game Theory, Natural Selection and Darwinian Dynamics, Cambridge University Press.

[18] J. von Neumann, Zur theorie der gesellschaftspiele, Mathematische Annalen, 100 (1928), pp. 295-320.

[19] J. von Neumann And O. Morgenstern, The Theory of Games and Economic Behaviour, Princeton University Press, Princeton, New jersey, USA, 1944.

[20] J. G. WARDROP, Some theoretical aspects of road traffic research, Proceedings of the Institution of Civil Engineers, (1952), pp. 325-378.

[21] J. WeIBUll, Evolutionary Game Theory, M.I.T. Press, Cambridge, U.S.A., 1995. 


\section{A E.S.S. and E.R.S.}

We recall the definition

Definition 4 A Wardrop equilibrium $p$ is said regular if there exists a $\Delta\left(X_{1}\right)$ neighborhood of $p$ such that over that neighborhood $q \mapsto H_{1}(x, q)$ is Lipshitz continuous for all $x \in X$ and the restriction of the quadrtic form $\left\langle r, H_{1}(q) r\right\rangle$ to measures $r$ orthogonal to $\mathbb{1}$ in $\mathcal{M}\left(X_{1}\right)$ is negative definite, both uniformly in $q$.

We prove here the theorem

\section{Theorem 3}

(i). All E.R.S. are E.S.S.

(ii). In the finite case, all regular Wardrop equilibria ${ }^{13}$ are E.R.S.

\section{Proof}

(i). Applying the E.R.S. condition to $q_{\varepsilon}=p+\varepsilon(q-p)$ and using the linearity of $F$ in its first argument, we get condion (3).

(ii). The proof of this point requires that there exist a compact set $K \subset \Delta(X)$ such that, for any positive $\bar{\varepsilon}$, the set $\left\{q_{\varepsilon}=p+\varepsilon(q-p), q \in K, \varepsilon \leq \bar{\varepsilon}\right\}$ be a neighborhood of $p$. (In particular, $p \notin K$.) It is why it is restricted to the finite case, where $\Delta(X)$ is isomorphic to the finite dimensional simplex.

Let $p$ be an E.S.S., $B(p) \in \Delta(X)$ the set of best responses $q$ to $p$, i.e. such that $F(q, p)=F(p, p), X_{1} \subset X$ its support, $G_{1}$ the restriction of $G$ to $X_{1} \times \Delta\left(X_{1}\right)$. Let also $q_{\varepsilon}$ and $K$ be as just said above. We recall the definition of the negative score function $\mathcal{E}(\varepsilon, q)=F\left(q-p, q_{\varepsilon}\right)$ and that $B(p)=\{q \mid \mathcal{E}(0, q)=0\}$, and that the derivative of $\mathcal{E}$ w.r.t. $\varepsilon$ is

$$
\mathrm{D}_{1} \mathcal{E}(\varepsilon, q)=\left\langle q-p, \mathrm{D} G\left(q_{\varepsilon}\right)(q-p)\right\rangle
$$

Consider first the case where $q \in B(p)$. Then $\mathcal{E}(0, q)=0$. Notice that

$$
\mathrm{D}_{1} \mathcal{E}(\varepsilon, q)=\left\langle q-p, \mathrm{DG}_{1}\left(q_{\varepsilon}\right)(q-p)\right\rangle
$$

where $p$ and $q$ are in $\Delta\left(X_{1}\right)=\Sigma_{n_{1}}$, and the scalar product is accordingly that of $\mathbb{R}^{n_{1}}$. As a consequence of the hypothesis of regularity, this derivative is negative forall $q \in K_{1}:=K \cap B(p)$. Since $K$ is compact, and therefore

\footnotetext{
${ }^{13}$ and all E.S.S. in the linear case, but this is proved in [8, Theorem 6.4.1]
} 
also $K_{1}, \mathrm{D}_{1} \mathcal{E}(0, q)$ is uniformly bounded away from 0 on $K_{1}$. Because the derivative is locally Lipshitz continuous w.r.t. $\varepsilon$, it follows that there exists a $\hat{\varepsilon}>0$ such that $\mathcal{E}(\varepsilon, q)<0$ for all $\varepsilon \in(0, \hat{\varepsilon})$, and because the Lipshitz constant is assumed to be uniform, this same $\hat{\varepsilon}$ is valid for every $q \in K_{1}$. Let us summarize :

$$
\exists \hat{\varepsilon}>0: \forall \varepsilon \in(0, \hat{\varepsilon}), \forall q \in K \cap B(p), \quad \mathcal{E}(\varepsilon, q)<0 .
$$

This can be stated with the help of the following concept:

Definition 5 Define the invasion barrier $\varepsilon_{0}(q)$ as

$$
\varepsilon_{0}(q)=\sup \left\{\varepsilon_{1} \in[0,1] \mid \forall \varepsilon \in\left(0, \varepsilon_{1}\right), \mathcal{E}(\varepsilon, q)<0\right\} .
$$

Then (16) reads $\forall q \in K_{1}, \varepsilon_{0}(q) \geq \hat{\varepsilon}>0$.

We claim the following:

Lemma 2 The function $\min \left\{\hat{\varepsilon}, \varepsilon_{0}(\cdot)\right\}$ is lower semi-continuous over $K$.

Proof of the lemma Let $\delta \in\left(0, \varepsilon_{0}(q)\right)$ be given. We want to prove that for every $q \in K$, there exists a neighborhood $\mathcal{N}(q)$ such that for $q^{\prime} \in \mathcal{N}(q)$, $\varepsilon_{0}\left(q^{\prime}\right) \geq \min \left\{\hat{\varepsilon}, \varepsilon_{0}(q)\right\}-\delta$, hence that

$$
\forall q^{\prime} \in \mathcal{N}(q), \forall \varepsilon \in\left(0, \min \left\{\hat{\varepsilon}, \varepsilon_{0}(q)\right\}-\delta\right), \mathcal{E}\left(\varepsilon, q^{\prime}\right)<0 .
$$

Assume first that $q$ and $q^{\prime}$ are in $B(p)$. We are in the situation above, $\varepsilon_{0}\left(q^{\prime}\right) \geq$ $\hat{\varepsilon}$, which proves the inequality.

If $q^{\prime} \notin B(p)$, which is always possible if $q \notin K_{1}$, then $\mathcal{E}\left(0, q^{\prime}\right)<0$, and by continuity and compacity, $\exists \varepsilon_{1}>0$ such that $\forall \varepsilon \in\left[0, \varepsilon_{1}\right], \mathcal{E}\left(\varepsilon, q^{\prime}\right)<0$. Also, $\exists \eta>0: \forall \varepsilon \in\left[\varepsilon_{1}, \varepsilon_{0}(q)-\delta\right], \mathcal{E}(\varepsilon, q)<-\eta$. Then, by continuity of $\mathcal{E}$ w.r.t. $q$, uniform w.r.t. $\varepsilon$, for $\mathcal{N}(q)$ small enough and $q^{\prime} \in \mathcal{N}(q), \mathcal{E}\left(\varepsilon, q^{\prime}\right)<0$. Hence we get the deisred result (17).

As a consequence of the lemma, $\varepsilon_{0}(q)$ has a positive minimum, say $\bar{\varepsilon}$ over $K$. It follows that

$$
\forall \varepsilon<\bar{\varepsilon}, \forall q \in K, \quad F\left(q, q_{\varepsilon}\right)<F\left(p, q_{\varepsilon}\right) .
$$

and hence by linearity of $F$ w.r.t. its first argument

$\forall \varepsilon \in(0, \bar{\varepsilon}), \forall q \in K, \quad F\left(q_{\varepsilon}, q_{\varepsilon}\right)-F\left(p, q_{\varepsilon}\right)=\varepsilon\left[F\left(q, q_{\varepsilon}\right)-F\left(p, q_{\varepsilon}\right)\right]<0$.

By definition of $K$, this set of $q_{\varepsilon}$ 's is a neighborhood of $p$.

This proves the theorem. 